Service social

\title{
Accompagner des personnes âgées en couple : un angle mort du travail social
}

\section{Corinne Dallera, Clothilde Palazzo-Crettol et Annick Anchisi}

Volume 61, numéro 1, 2015

Être reconnu et protégé

URI : https://id.erudit.org/iderudit/1033737ar

DOI : https://doi.org/10.7202/1033737ar

Aller au sommaire du numéro

Éditeur(s)

École de service social de l’Université Laval

ISSN

1708-1734 (numérique)

Découvrir la revue

Citer cet article

Dallera, C., Palazzo-Crettol, C. \& Anchisi, A. (2015). Accompagner des personnes âgées en couple : un angle mort du travail social. Service social, 61(1), 1-18.

https://doi.org/10.7202/1033737ar
Résumé de l'article

Actuellement, le nombre de personnes âgées vivant en couple et susceptibles de s'adresser à un service social augmente. Basé sur l'analyse qualitative de 13 entretiens avec des assistants et assistantes sociales accompagnant des personnes âgées et travaillant dans la partie francophone du Valais (un canton de la Suisse), cet article vise à explorer les questions suivantes : d'une part, comment la conjugalité est-elle prise en compte dans le cadre de

l'accompagnement social de personnes âgées? D'autre part, comment les inégalités de genre et les situations de couples conflictuelles sont-elles gérées ? Enfin, comment les prises de décision et les pratiques professionnelles face à ces situations sont-elles justifiées? 


\title{
Accompagner des personnes âgées en couple : un angle mort du travail social
}

\author{
DALLERA, Corinne \\ PALAZZO-CRETTOL, Clothilde \\ ANCHISI, Annick
}

\section{RÉSUMÉ}

Actuellement, le nombre de personnes âgées vivant en couple et susceptibles de s'adresser à un service social augmente. Basé sur l'analyse qualitative de 13 entretiens avec des assistants et assistantes sociales accompagnant des personnes âgées et travaillant dans la partie francophone du Valais (un canton de la Suisse), cet article vise à explorer les questions suivantes: d'une part, comment la conjugalité est-elle prise en compte dans le cadre de l'accompagnement social de personnes âgées? D'autre part, comment les inégalités de genre et les situations de couples conflictuelles sont-elles gérées ? Enfin, comment les prises de décision et les pratiques professionnelles face à ces situations sontelles justifiées?

Mots-clés : personnes âgées, conjugalité, couple, travail social, inégalité, genre, âgisme.

\section{ABSTRACT}

At present elderly couples are more likely to consult social services. This article is based on the qualitative analysis of 13 interviews with social workers supporting older people and working in the French-speaking area of Valais (a canton of Switzerland). It aims to explore the following questions: To what extent and how social workers take into account conjugality in elderly care? How do they deal with gender inequality and conflict situations in couple relationships? How do they justify their decisions and practices in front of those situations?

Key words: elderly, conjugality, couple, social work, inequality, gender, ageism. 


\section{INTRODUCTION}

En raison de l'allongement de l'espérance de vie et d'une amélioration globale de la santé fonctionnelle et psychique des personnes âgées (Lalive d'Epinay et Hofstetter-Bétemps, 2000 ; Lalive d'Epinay et Spini, 2008), un nombre de plus en plus important de personnes vivent en couple jusqu'à un âge avancé (Bonvalet et Lelièvre, 2005 ; Fux et al., 2006), même si ce phénomène est nettement plus courant pour les hommes que pour les femmes (Delbès, Gaymu, et Springer, 2006). En Suisse, $58 \%$ des hommes entre 80 et 84 ans vivaient en couple en 1980, ils étaient un peu plus de $69 \%$ en 2000. Pour les femmes, ce taux est passé de 17 à $24 \%$ (Höpflinger et Hugentobler, 2006). Cette tendance va s'accentuer à moyen terme pour les couples hétérosexuels, dans la mesure où les personnes qui atteignent actuellement un âge avancé appartiennent aux cohortes qui se sont mariées plus fréquemment que les cohortes antérieures et qui divorcent moins que les couples des cohortes ultérieures. On constate également que partout en Europe l'entrée en institution est de plus en plus tardive et qu'elle a lieu lorsque les personnes sont déjà fortement atteintes dans leur santé (Gérontologie et Société, 2005), un phénomène largement encouragé par des politiques de privatisation de l'aide et des soins de longue durée aux personnes âgées (Simonazzi, 2009). Vivre en couple permet de repousser le moment de l'entrée en institution, notamment pour les hommes en couple hétérosexuel, qui peuvent généralement compter sur le soutien d'une compagne plus jeune et dont l'espérance de vie est plus élevée (Delbès, Gaymu, et Springer, 2006). À l'âge de la grande vieillesse, la vie de couple pose cependant de grands défis en termes d'adaptation au moment des ruptures biographiques: placement d'un des conjoints en institution, entrée dans la démence, la maladie ou la dépendance, par exemple (Anchisi, 2008; Talpin et Joubert, 2008). Or, ces ruptures signifient le plus souvent la nécessité de recourir à une aide professionnelle. Comme celles et ceux de la santé, les professionnel.le.s de l'action sociale sont ainsi de plus en plus souvent amené.e.s à s'occuper de couples âgés.

Cet article présente une partie des résultats d'une recherche exploratoire et qualitative menée en 2012-2013 en Valais, un canton alpin et catholique de Suisse romande et portant sur la conjugalité de personnes âgées en couple hétérosexuel ${ }^{1}$ en lien avec des professionnel.le.s du travail social ${ }^{2}$. La question de recherche principale du projet était la suivante: Dans quelle mesure, l'interaction entre couples âgés et professionnel.le.s, performée par les rapports sociaux de sexe et la relation de travail social, modifie-t-elle les territoires du conjugal et à l'inverse dans quelle mesure cette interaction peut-elle transformer les usages professionnels ?

Dans le cadre de ce projet, des entretiens semi-directifs ont été menés avec 12 couples vivant à domicile $(\mathrm{N}=8)$ ou en établissements médico-sociaux $(\mathrm{EMS})^{3}(\mathrm{~N}=4)$ et avec des

1. Nous avons circonscrit notre objet de recherche aux pratiques professionnelles envers les couples hétérosexuels dans la mesure où s'intéresser aux pratiques des services sociaux envers tous les couples impliquerait de tenir compte de questions que nous n'abordons pas, comme les discriminations résultant de l'hétéronormativité de la prise en charge socio-sanitaire et qui constituerait en soi un objet de recherche.

2. Cette recherche intitulée «Vieillir ensemble sous le regard des professionnel·le·s : l'intimité théâtralisée ? 》 et menée sous la direction de Clothilde Palazzo-Crettol (HES-SO Valais-Wallis) et d'Annick Anchisi (HESAV) a été financée par le Fonds National Suisse de la recherche scientifique dans le cadre du programme DORE.

3. Les EMS accueillent des personnes souvent âgées présentant une ou plusieurs défaillances physiques, psychiques ou sociales, qui peuvent provoquer la dépendance, ou la perte d'autonomie. Ils correspondent aux EHPAD en France. 
professionnel.le.s du travail social, soit 13 assistants et assistantes sociales accompagnant des couples vivant à domicile et 4 animatrices socio-culturelles travaillant en EMS que ces couples sont amenés à rencontrer. En raison du fort taux de féminisation du secteur, seul un assistant social figurait parmi les professionnels et professionnelles interviewées dans le cadre de cette étude. Afin de respecter la réalité sexuée du terrain, mais également de préserver l'anonymat de l'interviewé, nous utilisons dans le texte qui suit le féminin pour l'ensemble des travailleurs et travailleuses sociales concernées. Les critères de sélection des professionnelles, qui étaient employées par des institutions partenaires de terrain durant la période où nous avons réalisés les entretiens, étaient la proximité qu'elles entretiennent avec les couples âgés. Par proximité, nous entendons, un contact professionnel relativement régulier et/ou durable. S'agissant des couples âgés, ils nous ont été signalés par les professionnelles sur des critères d'inclusion qui découlent de l'analyse de la littérature: l'âge, ainsi que l'entrée dans un processus de fragilisation caractérisée par l'intervention professionnelle et la possibilité de répondre à l'enquête. Notre échantillon de personnes âgées comprenait donc des couples vivant dans des lieux distincts (domicile et EMS) qui correspondent généralement à deux étapes du processus de fragilisation.

Deux grilles d'entretien distinctes - pour les professionnelles et les couples âgés - mais comprenant des parties communes ont été construites. Dans une perspective itérative (Olivier de Sardan, 2008), ces grilles ont été adaptées en fonction de l'avancée des travaux. Ainsi au fur et à mesure que nous avions des données sur les couples, nous avons affiné le questionnement destiné aux professionnelles et vice-versa. Afin de respecter les principes éthiques de la recherche, nous avons envoyé aux personnes susceptibles d'être interviewées une lettre d'information et un formulaire de consentement éclairé à remplir. Les entretiens ont duré environ une heure et demi. Concernant les professionnelles, les entretiens ont eu lieu dans leur bureau. Concernant les couples, chaque membre a été interviewé simultanément par deux chercheuses de l'équipe, dans deux espaces séparés de leur domicile ou de l'institution dans laquelle ils et elles résidaient, afin de garantir la confidentialité des propos recueillis.

À des fins d'analyse catégorielle, les entretiens ont été retranscrits in extenso. Puis, une lecture intégrale de tous les entretiens a été effectuée afin de mettre au jour les thèmes transversaux. Chaque entretien a ensuite été codé sur la base de ces thèmes à l'aide d'un logiciel libre de traitement qualitatif des données (TAMS Analyser). Des lectures et des recodages successifs ont ensuite permis d'affiner chaque code et ses sous-codes et de mettre au jour de nouveaux thèmes. Nous avons appliqué les règles habituelles d'anonymisation des données auxquelles nous avons ajouté des précautions supplémentaires, au vu du cadre d'interconnaissance dans lequel nous avons travaillé. Pour ce faire, nous avons modifié les variables contextuelles telles que l'âge, le nombre d'enfants, le métier, la nationalité - tant pour les professionnelles que pour les couples âgés -, dans la mesure où cela n'avait pas d'influence directe sur l'analyse. Au vu du nombre restreint d'entretiens réalisés dans le cadre de cette recherche exploratoire, nous avons également renoncé à effectuer une analyse par triade de personnes concernées par une situation (chaque membre du couple et la professionnelle qui l'accompagne), en vue de garantir l'anonymat des participant·e.s et cela malgré l'intérêt qu'aurait eu une telle démarche.

Cet article vise à explorer les questions suivantes dans une perspective compréhensive : d'une part, dans quelle mesure et comment des professionnelles d'un service social 
tiennent-elles compte du couple et de la conjugalité dans le cadre de l'accompagnement de personnes âgées en couple hétérosexuel? Et d'autre part, comment gèrent-elles les situations de couple inégalitaires ou conflictuelles? Quelles sont les «règles de conduites » face à ces situations, comment justifient-elles leurs prises de décision et leurs pratiques? Nous présentons dans un premier temps le cadre théorique de notre étude. Dans un deuxième temps, nous restituons la/les parole(s) des assistantes sociales interviewées. Enfin dans une troisième partie, nous présentons notre interprétation exploratoire des entretiens réalisés, en les contextualisant à partir de la notion d'accompagnement qui s'est imposée comme norme des bonnes pratiques dans le domaine du travail social depuis plus d'une vingtaine d'années. Nous nous sommes plus particulièrement intéressées aux rapports sociaux de sexe et d'âge.

Précisons encore que le texte qui suit se base sur l'analyse des entretiens menés avec les assistantes sociales, que nous analysons ici en tant que corpus homogène et indépendant des données recueillies dans le cadre des entretiens réalisés avec les couples et avec les animatrices socio-culturelles. Les assistantes sociales interviewées travaillaient dans trois types d'organisations: une organisation destinée spécialement aux personnes âgées $(\mathrm{N}=10)$, une ligue contre le cancer $(\mathrm{N}=1)$, et deux services d'aide et de soins à domicile $(\mathrm{N}=2)$. Toujours à des fins de garantir l'anonymat, nous avons également décidé de ne pas indiquer, dans cet article, le type d'organisation dans laquelle travaille l'assistante sociale dont nous citons un extrait d'entretien. Dans la suite de cet article, les assistantes sociales sont donc identifiées par un chiffre.

\section{CADRE THÉORIQUE}

\section{La prise en compte de la conjugalité dans l'action socio-sanitaire envers les personnes âgées}

La pluralité des acteurs et actrices impliquées dans la prise en charge des personnes âgées considérées en perte d'autonomie, et la complexité des rapports qui se construisent entre les familles, l'État, les associations et les professionnelles a été soulignée (voir notamment Weber, Gojard et Gramain, 2003). L'aide professionnelle arrive toujours après le soutien de la famille (Pennec, 2002, 2004; Bardet Blochet, 2005; Anchisi, 2008). Ainsi les professionnelles sont la plupart du temps face à des arrangements familiaux prédéfinis plus ou moins clairement (Höpflinger et Hugentobler, 2006). Or les rapports que les professionnelles entretiennent avec la famille et notamment le conjoint ou la conjointe ont une incidence sur les aspects techniques, relationnels et organisationnels de leur activité. Ces rapports peuvent notamment contribuer à augmenter ou diminuer la sympathie des professionnelles pour les bénéficiaires (Bouchayer, 2006). Höpflinger et Hugentobler (2006) soulignent, par ailleurs, à propos des soins à domicile qu'ils sont encore fortement axé sur l'individu. Ce constat nous semble pouvoir également s'appliquer à l'intervention sociale auprès de couples âgés. En effet, cette intervention s'inscrit dans les mutations qui transforment fortement le travail social depuis plusieurs années (voir par exemple: Ion, 2006 ; Aballéa, 2002 ; Bouquet, 2007), où l'individuation des prestations s'oppose à une vision plus globale. Ainsi, les interventions envers les familles demeurent centrées sur l'individu avec une réelle difficulté de penser un collectif (Modak et Palazzo, 2004). Pour cette recherche nous sommes parties de l'hypothèse qu'il en va de même pour les couples 
âgés, les professionnelles ayant peu d'outils pour penser les interdépendances et les inscriptions diverses des usagers et usagères (Chambers, 2004).

\section{Genre et vieillissement}

La gérontologie et les gender studies se sont longtemps mutuellement accusées de s'ignorer. Plusieurs auteur.e.s soulignent en effet que même si le sexe est fréquemment pris en considération dans les études gérontologiques, il s'agit encore souvent d'une simple variable ajoutée et que les rapports de genre se sont pas suffisamment théorisés ( $p$. ex. Katz et Maury, 2001 ; Russell, 2007 ; Krekula, 2007). Du côté des gender studies et des études féministes, le vieillissement a longtemps été négligé. Ce désintérêt peut notamment être attribué à la centralité accordée à la période de vie active et de la procréation, tant par les analyses de la division sexuelle du travail (Membrado, 2002 ; Krekula, 2007) que celles portant sur la famille (Krekula, 2007). Il est également relevé que la recherche féministe n'échappe pas à un certain âgisme (Katz et Maury, 2001 ; Membrado, 2013).

À la suite d'une série de travaux pionniers dans les années 1980 et 1990 (pour la Suisse, Stuckelberger et Höpflinger, 1996 ; Gognalons-Nicolet et Bardet Blochet, 1996, 1997), les recherches sur le vieillissement ont cependant progressivement développé des réflexions théoriques sur les rapports sociaux de sexe. Elles relèvent les doubles discriminations subies par les femmes âgées. Au niveau socio-économique notamment, les systèmes de retraite qui reproduisent les inégalités de genre de la vie professionnelle pénalisent les femmes au niveau financier (Stuckelberger et Höpflinger, 1996 ; Vielle, 2001 ; Bonnet, Buffeteau et Godefroy, 2004 ; Lewis, 2007) et les inscrivent pour les plus âgées en tout cas, dans un rapport de dépendance (Henchoz, 2008).

Le tournant des politiques sociales vers le maintien à domicile a également suscité un regain d'intérêt pour le vieillissement et les questions de genre dans les recherches sur les relations intergénérationnelles et les solidarités familiales (Membrado, 2002). Ces recherches, qui mettent en évidence le travail invisible fourni par les familles dans la prise en charge des personnes en perte d'autonomie, relèvent que ce travail, considéré comme improductif du point de vue salarié, est accompli essentiellement par des femmes, membres de la famille ou professionnelles (p. ex. Membrado, 2002 ; Pennec, 2002, 2004 ; GognalonsNicolet et Bardet Blochet, 1996, 1997 ; Bardet Blochet, 2005 ; Stark, 2005 ; Avril, 2003, 2008). En effet, en raison d'un processus de naturalisation du travail domestique et de soins en général, il « va de soi » que ce soit l'épouse dans le couple vieillissant, puis la fille et, en l'absence des deux premières, la bru, qui prenne en charge la personne âgée en perte d'autonomie. Ces études soulignent que les femmes de la famille, désignées ou autodésignées, expriment avec difficulté une grande solitude par rapport à ce lourd investissement, tant l'interdit est pesant (Membrado, 2002). Par ailleurs, dans bien des cas, les rapports sociaux de sexe influent sur la légitimité d'un soutien professionnel aux couples âgés, celui-ci étant plus fréquent du côté des époux que des épouses (Membrado, 2002). Aujourd'hui, les hommes sont toutefois de plus en plus souvent amenés à devoir assumer le rôle d'aidant et à s'y former (Höpflinger et Hugentobler, 2006), notamment au sein des classes populaires (Pennec, 2009, 2010).

Enfin la diffusion et la traduction d'écrits portant sur le care, notamment ceux de Gilligan (1986) et de leur critique par Tronto (2006), ont suscité de nouveaux questionnements au sein de la recherche féministe sur la prise en charge affective, curative ou éducative, tant 
familiale que professionnelle (voir par exemple pour la recherche francophone: Nouvelles Questions Féministes, 2004, Modak et Palazzo, 2008 ; Molinier, Laugier et Paperman, 2009). Ces analyses soulignent notamment que le care n'est pas une activité univoque. À l'empathie et à la sollicitude peuvent se combiner des sentiments rendus invisible par " l'idéologie de la tendresse féminine » et donc peu admis comme le dégoût et la haine (Molinier, 2004), desquels les représentations de la vieillesse et les pratiques des professionnelles peuvent se nourrir.

La perception d'une vieillesse dépréciée est attestée par la littérature (voir par exemple: Arfeux-Vaucher, 2001 ; Anchisi, Hugentobler et Luyet, 2006 ; Membrado, 2013) et analysée comme des représentations ${ }^{4}$ de l'altérité. En effet, « le vieux », c'est toujours l'autre, que l'on ait 20 ans ou passé 60 (Hummel et Tettamenti, 2008). Par ailleurs, ces représentations sont sexuées et les vieilles femmes accumulent les déficits (Perrig-Chiello, 2001). Pour Bozon, par exemple, la sexualité chez les tranches plus âgées de la population et particulièrement celle des femmes reste de l'ordre du tabou ou, en tout cas, celle qui est passée le plus sous silence et qui reste frappée par l'inégalité (Bozon, 2001, 2009). Ce tabou est également observable chez les professionnelles (Thibaud et Hanicotte, 2007 ; Ribes, Abras-leyral et Gaucher, 2007). Dans le même ordre d'idées, les analyses sur la violence conjugale ont pour cible des personnes de moins de 65 ans. Or, quelques entretiens exploratoires avec des assistantes sociales conduisent au constat que cette violence existe également dans les couples âgés, et qu'elle demeure particulièrement invisible dans la recherche, à l'exception de quelques études nord-américaines (pour une recension voir Charron, 2009, et pour un approfondissement, Montminy, 2005).

Ces éléments nous ont conduit à faire l'hypothèse que les perceptions professionnelles de la conjugalité des personnes âgées articulent au moins ces trois caractéristiques: décalées par rapport à la réalité des couples, négatives et genrées.

\section{RÉSULTATS}

\section{Des couples âgés révélés par la recherche}

Dès les premiers entretiens réalisés, un premier constat s'est imposé : malgré une présence plus marquée de couples âgés susceptibles de s'adresser à un service social, ceux-ci se dérobent la plupart du temps, "en tant que couple », aux regards des assistantes sociales que nous avons interviewées. Ces dernières ne les voient pas au sens propre, comme au sens figuré.

Dans leur pratique, elles ne voient, en effet, initialement souvent que l'un'e ou l'autre des partenaires, le ou la délégué.e aux affaires sociales qui s'adresse au service pour trouver de l'aide :

C'est le constat qu'on a fait : c'est, qu'en général, il y a une personne qui prend en charge la situation. C'est rare que j'aie eu deux personnes. Le couple qui est venu ensemble faire une demande, je n'ai pas trop le souvenir. (AS 6)

4. Nous insistons ici sur la fonction première des représentations sociales, qui est d'interpréter la réalité environnante en entretenant avec elle des rapports de symbolisation et en lui attribuant des significations (Guimelli, 1999). Par ailleurs, nous considérerons les représentations individuelles comme révélatrices du contexte social et culturel d'où elles émanent et qu'elles contribuent simultanément à révéler. 
Par ailleurs, la conjugalité à un âge avancé demeure également dans les marges, voir un impensé de l'accompagnement social tel qu'il est considéré par les assistantes sociales interviewées:

Pourtant je suis une vieille routinière. Eh bien, je pense que j'ai peu le souci du couple. [...]. Je ne me suis jamais posé la question : «comment prend-on en charge un couple âgé ? » (AS 13)

En soulignant une question que les assistantes sociales interviewées ne s'étaient jamais posée individuellement ou collectivement et qui n'avait jamais été thématisée au sein des institutions qui les emploient, les entretiens ont ainsi joué un rôle maïeutique. Le statut conjugal étant un paramètre qui définit les prestations dues dans le cadre des assurances sociales, même lors d'un accompagnement administratif simple, les assistantes sociales déploient bien une ou des pratiques d'accompagnement spécifiques envers une personne qui vit en couple.

\section{Être équitable face aux deux partenaires, tout en maintenant à distance la relation de couple}

Lorsqu'on leur demande à quoi elles doivent être attentives dans le travail social auprès de couples âgés, un élément fréquemment évoqué par les assistantes sociales que nous avons interviewées est de veiller à respecter une équité dans l'attention portée aux deux partenaires du couple, tant au niveau de l'écoute que de l'information donnée :

Moi, j'ai l'impression que ce n'est pas tellement différent d'une personne seule. Je dirais que le truc où il faut vraiment être attentive quand on intervient chez un couple, c'est que les deux puissent avoir la parole, parce qu'il y a souvent tendance à ce qu'il n'y en ait qu'un qui parle sur les deux, [cela arrive] quand même souvent. (AS 10)

Néanmoins, l'attention portée à la conjugalité ne va généralement pas au-delà de ce souci d'équité envers les deux partenaires (Boinon, 2011). La relation conjugale, quant à elle, considérée comme privée ou du ressort d'autres intervenant·e.s, est généralement reléguée hors du cadre professionnel de l'assistante sociale.

Afin d'éviter de s'impliquer ou de se trouver impliquées dans les rapports de couple, les assistantes sociales déploient une large palette de stratégies, allant de l'accompagnement prioritairement orienté sur l'individu à un subtil travail d'équilibriste, visant à tenir compte des deux partenaires, tout en maintenant à distance les questions qui relèvent de la relation conjugale :

S'il me demande une chose et que je peux répondre, comment il fonctionne le couple, moi, je ne regarde pas. (AS 6)

C'est vrai qu'en tout cas au niveau de la vie intime, à part ce que les gens me disent, je ne leur pose jamais la question. Donc je leur demanderais : "comment va votre mari, quelle est sa santé ? » Dans l'autre sens, «comment ça va entre vous? », je n'ai jamais posé la question. (AS 13)

Les citations qui précèdent témoignent de l'intégration de l'idée normative de la « bonne distance professionnelle » (da Silva, 2010), caractérisée par le maintien d'une stricte 
séparation entre privé et public et qui participe certainement aux nouvelles tensions vécues par le travail social (Savignat, 2009).

\section{Quand les « problèmes de couple " perturbent la relation d'accompagnement}

Contourner la question de la relation conjugale de leurs usagers et usagères n'est cependant pas toujours possible pour les assistantes sociales :

Eh bien là, je suis en plein dans une situation, je ne sais pas comment je vais m'en sortir. [...] Physiquement, lui ne peut plus rester à la maison, elle, elle ne peut pas s'isoler et elle a besoin de sortir, donc je dois trouver un compromis. [...] Donc, c'est trouver un lieu où lui est entouré et puis elle peut encore garder son réseau. Donc des clash, ça peut être ça, ça peut être vraiment des pleurs, des peurs aussi, de qu'est-ce que ça représente un EMS. [...] Mais ça peut être des clash, ça peut être vraiment des cris, ça peut être des choses assez brutales quand même, ou des gens qui ne se causent plus, ou alors qui ne se causent qu'à coups d'injures. (AS 7)

Des rapports inégalitaires ou conflictuels au sein du couple peuvent ainsi contraindre les assistantes sociales à devoir rompre avec le double principe d'équité envers les partenaires et de non-ingérence dans la relation de couple. C'est par exemple le cas lorsque l'un.e des deux se fait le seul et unique porte-parole du ménage. Face à ces situations, le souci de justice implique de rétablir dans une certaine mesure l'équilibre au sein du couple par une intervention, ou du moins une médiation dans la relation :

Disons qu'il y a des gens, quand ils viennent ou quand je vais chez eux, je pose la question à madame et c'est monsieur qui me répond. Au bout d'un moment, je dis : « Je m'excuse mais je m'adresse à madame, j'aimerais que ce soit elle qui me réponde. » (AS 7)

Le principe de non-ingérence, à l'inverse, suppose de considérer les rapports inégalitaires comme étant l'affaire du couple et donc d'accepter que les intérêts des deux parties ne soient pas pris en considération en toute équité :

Bon moi, j'ose pas trop par rapport à ça, je vais pas trop bousculer les choses. J'essaie que les gens arrivent à dire les choses qu'on m'a dites, mais par la personne elle-même. J'essaie. Je me dis : «si madame fait toujours la même plainte, si dans l'entretien, j'arrive », l'idée c'est qu'elle puisse le dire pour voir comment lui réagit. Ça, c'est l'idéal, mais c'est pas moi qui vais dire «madame m'a dit que ». En tout cas, moi, je le ferais pas. C'est ma façon de faire, mais je sais pas si c'est la bonne. (AS 6)

Comment les assistantes sociales orientent-elles alors leurs pratiques face à cette alternative ? Quel principe, celui de la non-ingérence ou celui de l'équité, va-t-il primer face à des couples perçus comme inégalitaires et comment les assistantes sociales justifientelles l'option retenue?

\section{Des pratiques professionnelles patinées par les rapports sociaux d'âge}

Au cours des entretiens réalisés, la plupart des assistantes sociales ont insisté sur le fait que leur mandat consistait avant tout à répondre aux demandes des usagers et usagères et non à leur imposer des mesures, comme l'illustrent les extraits ci-dessous : 
Comme je disais, nous, on ne peut faire que ce que le client nous demande de faire. Après, s'il y a quelque chose qui contrarie notre relation, eh bien, je vais le souligner, et puis si vraiment c'est un problème, je vais le souligner et puis je vais le re-souligner et puis je vais dire : "C'est un obstacle on ne peut pas continuer. » Ça peut arriver, mais on ne peut que proposer et après ils font ce qu'ils veulent. (AS 7)

On répond à la demande, on n'est pas un service qui va chercher d'autres choses. On essaie de voir s'il y a autre chose, mais si la personne ne nous donne pas plus d'information, on ne va pas aller la chercher, si on sent qu'il n'y a pas un gros problème. (AS 3)

Si presque aucune assistante sociale interviewée n'exprime explicitement un rejet du contrôle, une réserve quant au fait d'endosser le rôle de celle qui contrôle ou qui contraint est néanmoins bien perceptible dans la division du travail opérée dans les situations où elles ont estimé qu'une surveillance à des fins de protection de l'un-e des membres du couple ou qu'un placement en institution était nécessaire. Dans ces situations, l'intervention contraignante est en effet généralement déléguée à d'autres professionnelles, voire à la famille.

Dans le discours des assistantes sociales interviewées, cet attentisme revendiqué fonde sa légitimité dans le respect de la temporalité des usagers et des usagères, qui constitue également un principe leur permettant de distinguer leur mode d'intervention sur le terrain face à celui d'autres professionnelles.

Il y a ci, il y a ça, donc moi j'ai le sentiment que des fois ils ne veulent plus nous entendre et qu'ils nous ressentent comme des..., pas comme un soutien mais comme un poids de plus. Je vous sors ça comme ça, mais je l'ai exprimé des fois à mes collègues infirmières en disant « moi, je pense qu'on va trop vite, laissons venir les problèmes ». Alors c'est vrai que ce n'est pas très professionnel de dire ça, nous on les voit... Nous on sait, je veux dire on a tellement de situations qu'on sait. (AS 13)

Or le respect de la temporalité des usagers et usagères implique plus ou moins explicitement des pratiques différenciées selon l'âge de la part de certaines assistantes sociales interviewées.

Je pense que moi je manque beaucoup d'imagination face à des personnes âgées, alors que j'ai pas mal d'imagination dans des idées que je pourrais proposer ou des réponses que je fais aux gens - un peu déroutantes des fois - pour que ça bouge. II me semble qu'avec les personnes âgées, je me plains du côté statique qu'elles ont et puis je pense que je me mimétise à leur côté statique assez vite et puis, oui, assise dans ce salon qui est surchauffé.

(AS 5)

Mais, je dois être aussi honnête, c'est que moi j'ai un papa de 95 ans, qui a un sécutel ${ }^{5}$, qui a un certain nombre de choses qui se passent à la maison, des fois faciles, des fois moins faciles, et c'est vrai que je relativise tout le temps. C'est-à-dire, je pense qu'il a fait sa vie. II a eu même une vie assez heureuse. C'est pas le cas de toutes les personnes âgées. Mais je sais que j'ai tendance à relativiser ce qui arrive. Et la seule chose qui m'importe pour eux, c'est que financièrement ils aient ce à quoi ils ont droit. (AS 1)

5. Dispositif de sécurité téléphonique. 


\section{Des rapports sociaux de sexe invisibilisés par les représentations dominantes de la conjugalité à l'âge avancé}

Deux figures de couple emblématiques incarnent la conjugalité à l'âge avancé aux yeux des assistantes sociales interviewées. La première figure est celle d'une relation conjugale marquée par la dépendance en raison de maladie et/ou de handicap qui font que les intérêts, les projets, les objectifs de chaque partenaire ne sont plus les mêmes. La deuxième figure est celle d'une relation conjugale caractérisée par des différences fondamentales de statuts et de positions entre l'époux et l'épouse que la longévité du couple a fini par cristalliser.

Ces deux figures emblématiques, marquées par le déséquilibre, partagent selon les assistantes sociales une vision jusqu'au-boutiste du couple les rendant imperméables au changement et qui les distinguent des couples plus jeunes.

Des projets, je ne sais pas, mais en tout cas je trouve que le côté pour le meilleur et pour le pire, il est encore très fort. Alors de nouveau là aussi je pense qu'au niveau des générations, à mon avis, ça va changer. Mais je trouve que ces dames et ces messieurs, ils sont encore très « on s'est marié et on ira jusqu'au bout, même si on doit, entre guillemets, crever de fatigue, mais on ira jusqu'au bout, on fera tout ce qu'on peut, on a promis de faire tout ce qu'on peut ». Je trouve qu'au niveau de la promesse, pff... elle est très lourde. (AS11)

Toutefois, selon que l'asymétrie au sein de la relation conjugale est attribuée à la maladie/au handicap ou au « fonctionnement du couple », elle fera l'objet d'une attention et d'un « accompagnement » différencié de la part des professionnelles interviewées comme le souligne le tableau ci-dessous.

Tableau 1 :

Tableau comparatif des propos de trois assistantes sociales concernant les rapports conjugaux inégalitaires d'usagers et usagères

\begin{tabular}{|c|c|c|}
\hline & $\begin{array}{l}\text { Rapports inégalitaires attribués à la } \\
\text { maladie/handicap }\end{array}$ & $\begin{array}{l}\text { Rapports inégalitaires attribués au } \\
\text { " fonctionnement du couple » }\end{array}$ \\
\hline AS 11 & $\begin{array}{l}\text { C'est vrai que moi j'appuie aussi beaucoup } \\
\text { là-dessus quand je reçois l'aidant qui vient. } \\
\text { Eux, ils sont encore dans le meilleur ou le } \\
\text { pire, « on y va jusqu'au bout, on a promis ». } \\
\text { Moi, j'essaie de leur ouvrir une porte dans } \\
\text { qu'est-ce que c'est après, donc préparer } \\
\text { aussi l'après. Dans le sens où, si } 24 \text { heures } \\
\text { sur } 24 \text {, on est là parce qu'il y a le conjoint } \\
\text { qui est à la maison, qui a toujours besoin, le } \\
\text { jour où le conjoint part, alors que ce soit } \\
\text { qu'il parte définitivement ou qu'il parte en } \\
\text { EMS, ou hospitalisé, « qu'est-ce que vous } \\
\text { allez faire de votre journée ». }\end{array}$ & $\begin{array}{l}\text { Et puis c'est vrai qu'après en } \\
\text { m'expliquant l'histoire de famille, la sœur } \\
\text { m'explique aussi, c'est une situation } \\
\text { chronique, mais chronique depuis le jour } \\
\text { où ils se sont mariés. Donc, ce n'est pas } \\
\text { pour ça que je baisse les bras, on est bien } \\
\text { d'accord, mais c'est pour ça que je me dis } \\
\text { «j'observe de loin ». Je reste à } \\
\text { disposition, chaque fois que je vois } \\
\text { madame, je lui redis que je suis là à } \\
\text { disposition s'il y a quoi que ce soit. Mais } \\
\text { voilà, c'est un couple qui a vécu comme } \\
\text { ça toujours. }\end{array}$ \\
\hline AS 12 & $\begin{array}{l}\text { Maintenant que j'y pense, il y a peut-être } \\
\text { une difficulté. C'est vrai qu'il y a toujours un } \\
\text { qui est plus en forme que l'autre et ça, c'est } \\
\text { une chose que je garde en tête, c'est que si } \\
\text { tout à coup celui-là, il lui arrivait quelque }\end{array}$ & $\begin{array}{l}\text { Je dirais que madame parle beaucoup } \\
\text { mais monsieur la reprend tout le temps, } \\
\text { parce que c'est vrai que pour les choses } \\
\text { dont on parle, elle n'a pas beaucoup } \\
\text { d'idées par rapport aux papiers, aux }\end{array}$ \\
\hline
\end{tabular}




\begin{tabular}{|c|c|c|}
\hline & $\begin{array}{l}\text { Rapports inégalitaires attribués à la } \\
\text { maladie/handicap }\end{array}$ & $\begin{array}{l}\text { Rapports inégalitaires attribués au } \\
\text { «fonctionnement du couple » }\end{array}$ \\
\hline & $\begin{array}{l}\text { chose, ça deviendrait tout de suite } \\
\text { dramatique. Je ne pense même pas au } \\
\text { décès mais une hospitalisation ou une } \\
\text { chute ou quelque chose comme ça. } \\
\text { Intervieweuse : Donc ça, c'est une difficulté } \\
\text { que vous envisagez... } \\
\text { Oui }\end{array}$ & $\begin{array}{l}\text { assurances et tout ça. Mais oui, elle, elle } \\
\text { va tout le temps vouloir parler et puis lui } \\
\text { va lui dire : «mais non, c'est tout faux ». } \\
\text { Intervieweuse : /l corrige? } \\
\text { Oui } \\
\text { Intervieweuse: Et puis ça vous met mal à } \\
\text { l'aise, ça, des fois? } \\
\text { Non, c'est des gens que je connais bien. } \\
\text { Je vois que ça fonctionne comme ça. } \\
\text { C'est des gens qui s'entendent très bien } \\
\text { donc, malgré tout. C'est presque un peu } \\
\text { pour rire qu'il la reprend comme ça tout le } \\
\text { temps. }\end{array}$ \\
\hline AS 13 & $\begin{array}{l}\text { Quand c'est comme ça, je prends quand } \\
\text { même toujours le parti de l'aidant que j'ai } \\
\text { envie de vous dire, parce que je me dis, on } \\
\text { se dit, centrer autour de la personne âgée. } \\
\text { C'est la personne âgée qui est dépendante, } \\
\text { mais si l'aidant ne tient pas le coup, eh bien } \\
\text { la personne âgée, elle, n'a pas d'autre } \\
\text { choix que l'EMS ou une autre prise en } \\
\text { charge. }\end{array}$ & $\begin{array}{l}\text { Moi, je me dis, c'est leur histoire. Je veux } \\
\text { dire, s'ils ont fonctionné comme ça } \\
\text { pendant des années, mais de quel droit je } \\
\text { vais... Donc, j'aurais une écoute } \\
\text { empathique une fois pour l'un, une fois } \\
\text { pour l'autre. En règle générale, il y en a un } \\
\text { qui se plaint, l'autre on ne le voit pas et } \\
\text { puis voilà. }\end{array}$ \\
\hline
\end{tabular}

«L'épuisement de l'aidant » apparaît comme l'étalon à partir duquel les situations de couple «à risque » sont identifiées et observées à plus ou moins grande distance par les assistantes sociales interviewées. Ce point de vue cadré par différents programmes politiques et institutionnels ${ }^{6}$ semble en effet conditionner la manière dont les rapports de pouvoir au sein du couple sont pris en considération et le rôle que l'assistante sociale pourrait être amenée à jouer. La comparaison des propos des trois assistantes sociales, selon qu'elles évoquent l'une ou l'autre des situations montre que la naturalisation des situations est moins forte dans les situations liées au handicap que lorsque les rapports inégalitaires relèvent des rapports de domination des hommes sur les femmes et qui sont attribués sans questionnement au «fonctiomment du couple ». Ainsi, dans le premier cas, un futur est envisagé et « préparé » par les assistantes sociales, alors que, dans le second, le couple est «observé de loin » et les conséquences des rapports de pouvoir sont minimisés : «C'est presque un peu pour rire qu'il la reprend comme ça tout le temps. » Les représentations sociales plus spécifiques de la conjugalité à l'âge avancé, incarnées par les deux figures emblématiques que nous avons identifiées sur la base de nos entretiens, celle du couple dont le fonctionnement inégalitaire entre les sexes se serait cristallisé avec le temps et celle de la dépendance de l'un des membres du couple envers l'autre en raison de maladie et/ou de handicap semblent ainsi infléchir l'attentisme des assistantes sociales

6. À la suite de différents travaux de recherche soulignant le rôle essentiel des proches et notamment des femmes de la famille dans le maintien à domicile des personnes âgées (voir p. ex. : Bardet Blochet, 2005 ; Anchisi et Despland, 2010), différents programmes politiques et institutionnels en faveur des « proches aidants » ont été mis en place dans les cantons suisses romands au cours des dernières années visant à les reconnaître et les soutenir. Les enjeux en termes de genre et de classe de l'aide des proches dans le cadre du maintien à domicile ne sont toutefois que rarement pris en considération dans ces programmes. 
interviewée : vers l'immobilisme dans le premier cas, vers un peu plus d'interventionnisme dans le second.

\section{DISCUSSION}

En réponse à nos questions de départ, les résultats exposés ci-dessus montrent que la conjugalité ne constitue pas à première vue un critère déterminant dans le travail social envers les usagers et usagères tel que le conçoivent les assistantes sociales. En ce sens, leurs pratiques semblent cohérentes avec « une orientation professionnelle impulsée quasi exclusivement par le face-à-face travailleur social/usager » (Boinon, 2011, p. 135). Néanmoins, en jouant un rôle maïeutique, notre recherche a conduit les assistantes sociales à réfléchir sur leur posture et a révélé que penser à un couple demande une autre "parole professionnelle », un autre type de «négociation » et une nouvelle collaboration sur des «projets tant personnels qu'institutionnels » (Ladsous, 2007, p. 77). Deux principes notamment semblent guider les pratiques des assistantes sociales interviewées face aux couples âgés qu'elles accompagnent: le souci d'équité envers les deux partenaires et la non-ingérence dans la relation conjugale. Or, ces deux orientations ne sont pas toujours conciliables, notamment face à des relations de couple inégalitaires et/ou conflictuelles. Et, comme certain.e.s auteur.e.s l'ont montré à propos du travail social, elles relèvent de logiques différentes et parfois concurrentielles, à savoir: la «volonté transformatrice » qui redonne « aux personnes leurs places d'acteurs et leurs droits » (Bouquet, 2012, p. 24) et la « gouvernance biographique » (Bouquet, 2012, p. 25) qui accentue la psychologisation du travail social en attribuant la responsabilité de ce qui lui arrive aux qualités personnelles de l'individu (Buscatto, 2006 ; Chobeaux, 2006).

Les entretiens réalisés montrent également que, malgré un souci de soutenir l'aidant·e dans les cas de situation de dépendance de l'un ou l'autre des membre du couple, les pratiques des assistantes sociales interviewées demeurent tendanciellement attentistes, notamment lorsque les inégalités au sein du couple sont attribuées à une inégalité entre les hommes et les femmes, un «laisser-faire » qui est légitimé par les assistantes sociales interviewées par la notion de « ne pas faire pour, mais faire avec » les usagers et usagères. Comme le souligne Gaspar, la notion d'« accompagnement » s'est imposée dans le champ du travail social depuis les années 1990, tant dans les pratiques et les discours des travailleuses et travailleurs sociaux que dans la littérature savante et professionnelle, pour devenir « un mode de présentation légitime des pratiques de cet univers » (Gaspar, 2011, p. 1). Se positionnant en rupture avec les pratiques de prise en charge, les pratiques d'accompagnement seraient plus humaines et moins normatives, plus égalitaires et moins dominatrices, plus respectueuses des bénéficiaires et moins intrusives. La diffusion de la notion d'accompagnement a permis et permet notamment d'occulter les aspects liés au contrôle propre au travail social (Dubois, 2009 ; Bouquet, 2012), ce qui concourt également à garantir son succès. Le registre de l'accompagnement et notamment la fonction légitimatrice de l'expression « ne pas faire pour, mais faire avec » (Gaspar, 2011) semble constituer la matrice rhétorique de l'éthos professionnel (Fusulier, 2011) des assistantes sociales interviewées, le point d'ancrage de ce qu'elles considèrent être leurs (bonnes) pratiques.

Au-delà de cette contextualisation, les résultats de notre recherche semblent montrer que les pratiques d'accompagnement, le souci du «faire avec et non pas pour » n'est pas neutre, mais construit par les rapports sociaux d'âge et de genre. En effet, face à la 
contrainte à l'auto-activation des publics cibles plus jeunes, qui doivent désormais montrer et prouver qu'ils veulent s'en sortir au risque de sanctions (Dubois, 2009) ou l'empressement à signaler les enfants des classes populaires « en danger » (Serre, 2009), le relativisme et l'absence de perspective de changements proposée/assignée aux plus âgées, que laissent transparaître les entretiens menés dans le cadre de notre recherche, sont frappantes et en ce sens nous semblent témoigner d'un certain âgisme. Par ailleurs, les situations inégalitaires et de domination, les risques de violence conjugale ne sont pas considérés par les assistantes sociales interviewées de la même manière selon qu'ils sont perçus comme relevant d'un rapport de dépendance dû à la maladie ou au handicap et pouvant conduire à l'épuisement du ou de la conjoint.e (et restant donc d'une certaine manière du ressort de l'assistante sociale) ou s'ils sont attribués à des inégalités entre hommes et femmes qui seraient constitutives du fonctionnement « des couples de cette génération ». Confinés sans questionnement à une époque révolue, les rapports de domination domestique des hommes sur les femmes ne sont pas perçus comme des rapports socialement construits mais sont réduits à une forme de conjugalité archaïque et vouée à disparaître, qui serait spécifique aux «vieilles et aux vieux d'aujourd'hui », une marque en quelque sorte de leur altérité, ce qui leur vaut d'être relativisés et refoulés hors du champ de l'intervention professionnelle des assistantes sociales.

\section{CONCLUSION}

En raison de son caractère exploratoire, les résultats de cette recherche ne peuvent pas être généralisés. Malgré leurs limites, ils nous semblent néanmoins corroborer des études menées dans d'autres contextes. En effet, ils reflètent selon nous une certaine normativité professionnelle, construite ici autour du concept d'accompagnement, à la fois peu susceptible de «dégenrer la famille » (Modak, Messant et Keller, 2013, p. 70) et soumise pour les plus âgé.e.s au pouvoir « d'aimantation » de la notion de dépendance (Amyot, 2012, p. 128), et cela, malgré une réelle volonté de soutien et de respect de l'équité envers les deux membres du couple de la part des assistantes sociales interviewées. Par ailleurs, ces deux aspects sont intimement liés dans la mesure où les représentations dégradées de la vieillesse et notamment celle de la dépendance invisibilisent les rapports de genre. Dans cette perspective, le développement de recherches portant plus spécifiquement sur l'articulation des rapports sociaux de sexe et d'âge dans le travail social nous semblerait particulièrement utile et prometteur. Dans la même perspective, une réflexion intégrant les rapports sociaux dans le cadre des mesures et programmes visant à encourager le maintien à domicile des personnes âgées en perte d'autonomie nous semble essentielle afin d'éviter la reproduction des inégalités sociales et notamment de genre.

DALLERA, Corinne

Chargée de recherche à l'EESP

Haute école de travail social et de la santé Lausanne, Suisse

PALAZZO-CRETTOL, Clothilde

Professeure

Haute Ecole de Travail Social HES-SO Valais/Wallis 
ANCHISI, Annick

Professeure

Haute Ecole de santé Vaud (HESAV), HES-SO

Lausanne, Suisse.

\section{BIBLIOGRAPHIE}

Aballéa, F. (2002). «Travail social et travailleurs sociaux : le divorce ? », Recherche sociale, $n^{\circ} 163$, p. 16-31.

Amyot, J.-J. (2012). « Vieillesse, contrôle social et idéologie sécuritaire. Entre autonomie et dépendance », Vie sociale, $n^{\circ} 1$, p. 125-143.

Anchisi, A. (2008). « De parent à résidant, le passage en maison de retraite médicalisée », Retraite et Société, Familles et professionnels auprès des personnes dépendantes, vol. $1, \mathrm{n}^{\circ} 53$, p. $167-182$.

Anchisi, A., et B. Despland (2010). «Les proches d'un parent âgé dépendant : le cœur du dispositif de l'aide et des soins à domicile », dans A. Blanc (dir.), Les aidants familiaux. Grenoble, Presses Universitaires de Grenoble.

Anchisi, A., V. Hugentobler et V. Luyet (2006). Placer une personne âgée démente en EMS, perspectives familiales et soignantes, Zurich, Cahiers de la Société Suisse pour la Politique de la Santé, $n^{\circ} 87$.

Arfeux-Vaucher, G. (2001). « Images de la vieillesse dans la littérature enfantine et auprès des jeunes enfants », Retraite et société, n 34, p. 89-111.

Avril, C. (2003). «Les compétences féminines des aides à domicile », dans F. Weber, S. Gojard et A. Gramain (dir.). Charges de famille. Dépendance et parenté dans la France contemporaine, Paris, La Découverte.

Avril, C. (2008). «Les aides à domicile pour personnes âgées face à la norme de sollicitude », Retraite et société, n 53, p. 49-65.

Bardet Blochet, A. (2005). "Le travail invisible des femmes auprès des proches âgés », dans Geschlecht und Gesundheit in den verschiedenen Lebensaltern. Aktuelle Forschungsarbeiten aus dem Bereich Gender Health / Genre et santé aux différents âges de la vie. Recherches actuelles dans le domaine Gender Health (Documentation du $3^{e}$ colloque national du réseau de recherche Gender Health), Brugg, Forschungsnetzwerk Gender Health.

Boinon, C. (2011). « La confrontation de l'éthique du travail social aux principes de justice sociale », Vie sociale, $n^{\circ} 3$, p. 133-149.

Bonnet, C., S. Buffeteau et P. Godefroy (2004). « Retraites, vers moins d'inégalités entre hommes et femmes ? », Population et Sociétés, n 401.

Bonvalet, C., et E. Lelièvre (2005). « Relations familiales des personnes âgées », Retraite et société, $\mathrm{n}^{\circ} 45$, p. 43-67.

Bouchayer, F. (2006). « Soigner des personnes âgées : quels effets sur les professionnels de la santé », Revue suisse de sociologie, vol. 32, n 3, p. 457-474. 
Bouquet, B. (2007). «Le travail social à l'épreuve. Face aux défis, dynamiques et reconquête de sens... », Empan, vol. 68, n 4, p. 35-42.

Bouquet, B. (2012). «Analyse critique du concept de contrôle social. Intérêts, risques et limites », Vie sociale, $n^{\circ} 1$, p. 15-28.

Bozon, M. (2001). «Sexualité et conjugalité », dans T. Blöss (dir), La dialectique des rapports hommes-femmes, Paris, Presses universitaires de France.

Bozon, M. (2009). Sociologie de la sexualité, collection «Domaines et approches », $2^{\mathrm{e}}$ éd., Paris, Armand Colin.

Buscatto, M. (2006). «Des hiérarchies intermédiaires sous le signe de la "psychologisation sociale" », Empan, n 61, p. 69-75.

Chambers, P. (2004). «The case for critical social gerontology in social work education and older women », Social Work Education, vol. 23, nº 6, p. 745-758.

Charron, F. (2009). La violence faite aux femmes aînées francophones. Une problématique à cerner, des services à offrir. Rapport fait à Action ontarienne contre la violence faite aux femmes.

Chobeaux, F. (2006). «Y a-t-il une psychologisation des formations du social ? » Vie sociale et traitements, vol. 91, $\mathrm{n}^{\circ} 3$, p. 49-50.

da Silva, E. (2010, septembre). La posture professionnelle des assistantes sociales : quand l'éthique de la distance masque le contôle de la conformation de genre. Communication présentée au colloque « La dimension relationnelle des métiers de service : Cache-sexe ou révélateur du genre ? », Université de Lausanne, récupéré le 26 novembre 2014 du site du Centre en étude genre LIEGE :

http://www.unil.ch/files/live//sites/liege/files/shared/TexteEmmanuelDaSilva.pdf.

Delbès, C., J. Gaymu et S. Springer (2006). «Les femmes vieillissent seules, les hommes vieillissent à deux. Un bilan européen », Population et Sociétés, nº 419.

Dubois, V. (2009). « Le paradoxe du contrôleur. Incertitude et contrainte institutionnelle dans le contrôle des assistés sociaux », Actes de la recherche en sciences sociales, $\mathrm{n}^{\circ} 178$, p. 28-49.

Fusulier, B. (2011). «Le concept d'ethos. De ses usages classiques à un usage renouvelé », Recherches sociologiques et anthropologiques, vol. 42, n 1, p. 97-109.

Fux, B., C. Sauvain-Dugerdil, H. Stutz, S. Strub, A. Leuba, C. Tritten et J. Krummenacher (2006). Soigner, garder et payer. La famille et les phases tardives de la vie. Berne, Commission fédérale de coordination pour les questions familiales (COFF).

Gaspar, J.-F. (2011, août-septembre). Métamorphose d'une pratique "émancipatrice » en pratique de rationalisation économique : l'accompagnement en travail social. Version de travail de la communication pour le $11^{\mathrm{e}}$ Congrès de l'Association française de science politique Section thématique 20 : «La managérialisation de l'État social : le cas de la France dans le contexte international ». Récupéré le 11 novembre 2014 du site de l'ASFP : http://www.afsp.info/congres2011/sectionsthematiques/st20/st20gaspar.pdf.

Gérontologie et Société (2005). « L'entrée en institution », n 112. Paris, Fondation nationale de gérontologie. 
Gilligan, C. (1986). Une si grande différence, Paris, Flammarion.

Gognalons-Nicolet, M. (dir.) (1997). Genre et santé après 40 ans. La santé des hommes et des femmes dans la seconde partie de vie, Berne, Hans-Huber.

Gognalons-Nicolet, M., et A. Bardet Blochet (1996). «Vieillir au féminin, vieillir au masculin », Gérontologie et Société, n 77 , p. 68-78.

Guimelli, C. (1999). La pensée sociale, Paris, Presses universitaires de France.

Henchoz, C. (2008). «Le rôle de l'indépendance financière dans la construction du lien conjugal contemporain. L'analyse du processus d'individualisation des finances dans trois générations de couples » [en ligne], Enfances, Familles, Générations, nº 9, p. 36-51.

Höpflinger, F., et V. Hugentobler (2006). Soins familiaux, ambulatoires et stationnaires des personnes âgées en Suisse, Genève, Médecine \& Hygiène (Observatoire suisse de la santé).

Hummel, C., et M. Tettamanti (2008). « Le vieux est un autre », dans Au fil du temps. Le jeu de l'âge, Lausanne et Gollion, Fondation Claude Verdan et Infolio.

Ion, J. (2006). Le travail social au singulier : la fin du travail social ?, Paris, Dunod, 2006.

Katz, S., et H. Maury (2001). «Les vieilles dames de Charcot », Cahiers du Genre, vol. 31, $\mathrm{n}^{\circ} 2$, p. $105-128$.

Krekula, C. (2007). «The Intersection of Age and Gender. Reworking Gender Theory and Social Gerontology », Current Sociology, vol. 55, n² 2, p. 155-171.

Ladsous, J. (2007). «Posture du corps et de l'esprit », Vie sociale et traitements, $\mathrm{n}^{\circ}$ 96, p. 74-77.

Lalive d'Épinay, C., J.-F. Bickel, C. Maystre et N. Vollenwyder Nathalie (2000). Vieillesses au fil du temps, 1979-1994: une révolution tranquille: santé, situations de vie, formes de participation et visions du monde des personnes âgées en Suisse. Lausanne, Réalités sociales

Lalive d'Epinay, C., et D. Spini (dir.) (2008). Les années fragiles. La vie au-delà de quatrevingts ans. Québec, Presses de l'Université de Laval.

Lewis, J. (2007). "Gender, ageing and the "new social settlement": the importance of developing a holistic approach to care policies », Current sociology, vol. 55, n ${ }^{\circ} 2$, p. 271 286.

Membrado, M. (2002). "L'aide à la vieillesse à l'épreuve des rapports sociaux de sexe », dans N. Le Feuvre (dir.), "Le genre: de la catégorisation du sexe », UTINAM, Revue d'anthropologie et de sociologie, $\mathrm{n}^{\circ}$ 5, p. 151-172.

Membrado, M. (2013). « Le genre et le vieillissement : regard sur la littérature », Recherches féministes, vol. 26, $\mathrm{n}^{\circ} 2$, p. 5-24.

Michel, M. (2013). « De l'éducation à l'accompagnement, quelles questions pour le travail social », Vie sociale et traitements, $n^{\circ} 4$, p. 177-190. 
Modak, M., et C. Palazzo (2004). Rapport à l'intention des membres du groupe "Mineurs II » de l'office du tuteur général. Étude exploratoire des dossiers de recherche en paternité, période 1980-2003. Rapport non publié.

Modak, M., de Kinkelin, C. et N. Benelli (2008). « La reconnaissance du care dans le travail social: éléments pour une analyse du travail des assistant.e.s sociaux » dans $M$. Rosende et N. Benelli (dir.), Laboratoires du travail, Lausanne, Antipodes.

Modak, M., Messant, F. et V. Keller (2013). "Les normes d'une famille "juste" dans les interventions des assistantes et assistants sociaux de l'aide sociale publique », Nouvelles Questions Féministes, vol. 32, n² 2, p. 57-72.

Molinier, P. (2004). « La haine et l'amour, la boîte noire du féminisme ? Une critique de l'éthique du dévouement », Nouvelles Questions Féministes, vol. 23, n 3, p. 12-25.

Molinier, P., S. Laugier et P. Paperman (2009). Qu'est-ce que le care ? Soucis des autres, sensibilité, responsabilité, Paris, Payot.

Montminy, L. (2005). « Older women's experiences of psychological violence in their marital relationships », Journal of Gerontological Social Work, vol. 46, n² 2, p. 3-22.

Nouvelles Questions Féministes (2004). «Famille-Travail : Une perspective radicale », vol. $23, n^{\circ} 3$.

Olivier de Sardan, J.-P. (2008). La rigueur du qualitatif. Les contraintes empiriques de l'interprétation socio-anthropologique, Louvain-la-Neuve, Academia-Bruylant.

Pennec, S. (2002). «La politique envers les personnes âgées dites dépendantes: providence des femmes et assignation à domicile », « Le genre des politiques publiques: des constats et des actions », Lien social et Politiques, $n^{\circ} 47$, p. 129-142.

Pennec, S. (2004). «Les configurations filiales face au vieillissement des ascendants », Empan, vol. 52, $\mathrm{n}^{\circ}$ 1, p. 86-94.

Pennec, S. (2009). «Le soutien des fils envers leurs parents âgés ». Réciproques, vol. 1, p. $91-103$.

Pennec, S. (2010). «Les configurations de soin envers les parents âgés. Des différences selon le genre, les milieux sociaux et la part des services professionnels », dans G. Cresson et M. Mebtoul (dir.), Famille et santé, Paris, Presses de l'École des hautes études en santé publique, p. 31-62.

Perrig-Chiello, P. (2001). «Images sexuées de la vieillesse ». Retraite et société, $\mathrm{n}^{\circ} 34$, p. 69-87.

Ribes, G., K. Abras-leyral et J. Gaucher (2007). « Le couple vieillissant et l'intimité ». Gérontologie et société, $\mathrm{n}^{\circ}$ 122, p. 41-62.

Russell C. (2007). «What Do Older Women and Men Want? Gender Differences in the 'Lived Experience' of Aging », Current Sociology, vol 55, n² 2, p. 173-192.

Savignat, P. (2009). « Le travail social aux défis du néolibéralisme. Entre le et la politique. » Le sociographe, vol. $30, n^{\circ} 3$, p. 21-29.

Serre, D. (2009). Les coulisses de l'Etat social. Enquête sur les signalements d'enfants en danger. Paris, Raisons d'agir. 
Simonazzi, A. (2009). "Care regimes and national employment models », Cambridge Journal of Economics, vol. 33, $\mathrm{n}^{\circ} 2$, p. 211-232.

Stark, A. (2005). «Warm Hands in Cold Age - On the Need of New World Order of Care ». Feminist Economics, vol. 11, n 2, p. 7-36.

Stuckelberger, A., et F. Höpflinger (1996). Vieillissement différentiel : hommes et femmes, Zürich, Seismo.

Talpin, J. M., et C. Joubert. (2008). « Vieillissement du couple, vieillissement dans le couple et séparation », Cahiers de psychologie clinique, vol. 31, n² 2, p. 107-134.

Thibaud, A., et C. Hanicotte (2007). «Quelles représentations les soignants ont-ils de la sexualité des sujets vieillissants ? ». Gérontologie et société, n 122, p. 125-137.

Tronto, J. (2009). Un monde vulnérable. Pour une politique du care, Paris, La Découverte.

Vielle, P. (2001). La sécurité sociale et le coût indirect des responsabilités familiales, Bruxelles, Bruylant.

Weber, F., S. Gojard et A. Gramain (dir.). (2003). Charges de famille. Dépendance et parenté dans la France contemporaine, Paris, La Découverte. 